\title{
Sleep studies and supportive ventilatory treatment in patients with congenital muscle disorders
}

\author{
Y Khan, J Z Heckmatt, V Dubowitz
}

\begin{abstract}
Eight ambulant children aged 6-13 years, four with congenital myopathy, two with congenital muscular dystrophy and two with the rigid spine syndrome, presented with recurrent chest infections, morning headaches, shallow breathing at night, or respiratory failure. Polysomnography confirmed the presence of nocturnal hypoxaemia with oxygen saturation on average less than $90 \%$ for $49 \%$ of sleep and less than $80 \%$ for $19 \%$ of sleep accompanied with severe hypoventilation. Additionally there was sleep disturbance characterised by an increased number of wake epochs from deep sleep (in comparison to 10 non-hypoxaemic subjects). The severity of sleep hypoxaemia did not correlate with symptoms. Treatment with night time nasal ventilation was started and repeat polysomnography showed normal overnight oxygen saturation and a reduced number of wake epochs during deep sleep. It is important to be vigilant for sleep hypoventilation in these patients and sleep studies should be part of the routine respiratory evaluation. Treatment with nasal ventilation is effective in reversing the nocturnal respiratory failure without significant disturbance to life style.

(Arch Dis Child 1996; 74: 195-200)
\end{abstract}

Keywords: myopathy, polysomnography, ventilation.

Sleep hypoxaemia associated with chronic respiratory failure is a recognised complication of congenital muscle disorders. ${ }^{1-5}$ The risk of respiratory problems varies and is high in congenital muscular dystrophy and certain types of congenital myopathies. Treatment is particularly worthwhile when the hypoxaemia occurs in association with mild physical disability. ${ }^{3}$ The recent introduction of non-invasive night time intermittent positive pressure ventilation by nasal mask (nasal ventilation) allows much easier management than was previously possible with the cuirass, iron lung, or tracheotomy. There is less disturbance of lifestyle as the equipment is compact, portable, relatively quiet, and easy to use. ${ }^{5-11}$ Despite the advantages of early diagnosis there have been few detailed polysomnographic studies in these patients and there is little information on the pathophysiology and diagnostic features of the hypoxaemia.

In this paper we describe the effect of treatment on a series of children with congenital myopathy who presented with sleep hypoventilation. We describe an approach to diagnosis that allows early detection and management of these patients and study of the effects of ventilation on sleep, respiratory function, and patient well being.

\section{Methods}

PATIENTS

We studied eight children (four girls and four boys) who presented to the muscle clinic at the Hammersmith Hospital (table 1). The mean (range) age at presentation was 10 (6-14) years. Despite having a severe respiratory problem, general disability was mild and all the patients were ambulant.

Their clinical presentation was as follows (table 1). Two sisters with congenital muscular dystrophy (patients 1 and 2) were reported to have shallow breathing and cyanosis at night, but without symptoms or history of chest infection. The other six patients had a history of recurrent chest infection (usually requiring hospital admission), four of these had morning headaches, three required supplementary oxygen, and two were on treatment for cor pulmonale. One (patient 6) was referred for routine evaluation of respiratory function in preparation for scoliosis surgery without a breathing problem being suspected. The youngest patient (5), who had nemaline myopathy, presented in acute respiratory failure during a chest infection. She had not previously had respiratory decompensation and her daytime gases were normal. Previous sleep studies had shown increasing sleep hypoxaemia, but up until that time she had been too young to tolerate nasal ventilation and cuirass ventilation was not successful. In general, the importance of the patients' symptoms and the underlying respiratory problems were underestimated.

The main indication for treatment was symptoms and unequivocal evidence of hypoxaemia either on the basis of daytime blood gases or sleep study. The most important symptoms were chest infections and morning headaches. All the patients reported daytime lethargy and fatigue and only one patient (6) was able to attend full time education. Three others (patients 4, 7, and 8) attended school part time but the remaining five received home tuition. In the eight patients there had been a total of nine prolonged admissions for chest infection during the preceding year, which in one patient lead to acute respiratory failure requiring ventilation. There were also symptoms of poor appetite and poor weight gain. Four patients were below the 3 rd centile for weight, three were on the 10th centile, and one was on the 25 th. 
Table 1 Diagnostic features and effect of night time nasal ventilation

\begin{tabular}{|c|c|c|c|c|c|c|c|c|}
\hline & \multicolumn{8}{|c|}{ Patient No } \\
\hline & 1 & 2 & 3 & 4 & 5 & 6 & 7 & 8 \\
\hline Sex & F & $\mathbf{F}$ & $\mathbf{M}$ & $\mathbf{M}$ & $\mathbf{F}$ & F & $\mathbf{M}$ & $\mathbf{M}$ \\
\hline Age (years) & 13 & 7 & 9 & 11 & 6 & 12 & 14 & 10 \\
\hline Ambulant & + & + & + & + & + & + & + & + \\
\hline Diagnosis* & CMD & CMD & $\mathbf{M}$ & $\mathbf{N}$ & $\mathbf{N}$ & $M C^{\$}$ & RS & RS \\
\hline Underbreathing reported & + & + & - & - & - & - & + & + \\
\hline Cyanosis reported & + & + & + & - & - & - & + & + \\
\hline Chest infections & - & - & + & + & + & + & + & + \\
\hline Morning headaches & - & - & + & + & - & + & + & + \\
\hline Acute respiratory failure & - & - & + & - & + & - & - & - \\
\hline Cor pulmonale & - & - & + & - & - & - & + & - \\
\hline Daytime oxygen & - & - & + & - & - & - & + & + \\
\hline Night time oxygen & - & - & + & - & - & - & + & + \\
\hline Scoliosist & ++ & + & ++ & + & + & ++ & + & - \\
\hline Spinal fusion & + & - & NP & - & - & + & + & - \\
\hline Abdominal squeeze & + & - & + & - & - & + & + & + \\
\hline Abdominal paradox & + & + & - & - & - & - & + & + \\
\hline Vital capacity (\% expected) & 27 & 56 & 17 & 50 & - & 40 & 45 & 17 \\
\hline \multicolumn{9}{|c|}{ Daytime arterial oxygen tension $(\mathrm{kPa}) \ddagger$} \\
\hline Before ventilation & $8 \cdot 6$ & $12 \cdot 5$ & $\begin{array}{r}6.9 \\
10.6\end{array}$ & 12 & 11 & $\begin{array}{r}8 \cdot 6 \\
11.3\end{array}$ & $11 \cdot 3 \delta$ & $14 \cdot 5 \S$ \\
\hline \multicolumn{9}{|c|}{ Daytime arterial carbon dioxide tension $(\mathrm{kPa}) \ddagger$} \\
\hline Before ventilation & $8 \cdot 5$ & $5 \cdot 1$ & $5 \cdot 6$ & $6 \cdot 6$ & 6 & $4 \cdot 1$ & $7 \cdot 3$ & $6 \cdot 8$ \\
\hline After ventilation & $6 \cdot 7$ & $5 \cdot 0$ & $5 \cdot 3$ & $5 \cdot 0$ & $5 \cdot 1$ & $4 \cdot 5$ & $6 \cdot 2$ & 5 \\
\hline \multicolumn{9}{|c|}{ Oxygen saturation $<90 \%$ as $\%$ of total sleep } \\
\hline On diagnostic sleep study & 99 & 15 & 40 & 14 & 20 & 100 & 66 & 45 \\
\hline After nasal ventilation & 0 & 0 & 0 & 0 & 0 & 2 & 0 & 0 \\
\hline
\end{tabular}

${ }^{\star} \mathrm{CMD}=$ congenital muscular dystrophy, $\mathrm{M}=$ minicore myopathy, $\mathrm{N}=$ nemaline myopathy, $\mathrm{MC}=$ 'minimal change' myopathy, $\mathrm{RS}=$ rigid spine syndrome with dystrophic change on needle muscle biopsy.

$t+\mathrm{Cobb}$ angle up to $40^{\circ},++\mathrm{Cobb}$ angle $>40^{\circ}$. NP = surgery for scoliosis was not possible.

‡Values at diagnosis of nocturnal hypoventilation before treatment and after regular ventilation established.

SSupplementary oxygen at the time of the test.

\$This patient was referred for routine respiratory evaluation before scoliosis surgery.

Six patients had abnormal respiratory muscle activity, either abdominal paradox when lying supine or an abdominal 'squeeze' manoeuvre in the erect position, that is, contraction of the abdominal muscles in expiration, ${ }^{3}$ or both. Seven had scoliosis, severe in three, three of whom received subsequent surgical stabilisation. ${ }^{12}$ Two patients had the rigid spine syndrome in association with a histological picture of muscular dystrophy on biopsy. All patients had daytime arterial blood gases while at rest breathing air (except where supplementary oxygen was required as specified in table 1). This was repeated as necessary once ventilation was established. Vital capacity was measured with a portable spirometer (Micromedical, Rochester, Kent) and compared with normal values. ${ }^{13}$

\section{SLEEP STUDIES}

Sleep studies were carried out on a relatively quiet section of the open ward. Our practice was to use overnight oximetry as an initial screen, followed by non-invasive polysomnography (diagnostic sleep study). We used the Oxford Medilog Multiparameter Recorder (Oxford Medical Ltd) which recorded eight channels of information: (1) electroencephalogram (EEG) channel C4-A1, (2 and 3) bilateral electro-oculogram, (4) submental electromyogram, (5 and 6) thoracoabdominal movements by inductance plethysmography ('Respibands', Ambulatory Monitoring Inc, Ardsley, NY), (7) air flow by an oronasal thermistor, and (8) arterial oxygen saturation (Ohmeda Biox 3700). Although patients had usually had one night of oximetry in the hospital before the sleep study, there was no acclimatisation night to the polysomnography. The recorder was small, portable, and did not disrupt ward routine. The oximetry data reported was from the polysomnography night.

We replayed the recordings on a computerised Medilog 9000-111 system. We analysed respiration during hypoxaemic episodes from printouts via a modified eight channel EEG Mingograph. The sleep recordings and oximetry were automatically analysed using the Medilog (SS-90 software version 4.82) sleep stager and also visually analysed.

For our analysis we divided sleep into 'light sleep' (stages 1 and 2), 'deep sleep' (stages 3 and 4), and rapid eye movement (REM) sleep. ${ }^{14}$ We analysed measures indicative of sleep quality, namely: sleep efficiency, duration of REM sleep, and the period spent awake after the initial onset of sleep. ${ }^{14} 15$ The latter was further subdivided into the number of times the patient had a 30 second or greater awake epoch after each stage of sleep (wake shifts). ${ }^{16}$ A 30 second awake epoch was defined as the development of alpha and/or low voltage mixed frequency activity on the EEG. ${ }^{17}$ Sleep efficiency was defined as the percentage of the actual sleep time divided by the total recording time. ${ }^{15}$ Sleep architecture was defined as the cyclical alteration of REM and non-REM sleep throughout the night. ${ }^{18}$ The duration of hypoxaemia in deep and REM sleep were quantified from the hypnogram.

The detailed respiratory data allowed breath to breath analysis of airflow, chest and abdominal movements. Although these data were not quantifiable, a diminution of breath or airflow amplitude was taken as a reduction in respiration. We classified apnoeas and periods of hypoventilation lasting longer than 10 seconds according to the criteria of Cherniak. ${ }^{19}$

\section{ARTIFICIAL VENTILATION}

After the diagnostic sleep study the patients were started on night time nasal ventilation 
breathing air. They used the BiPAP S/T ventilator (Respironics, USA) - that is, biphasic positive airways pressure. The ventilator is lightweight, compact, and portable with adjustable voltage power supply.

Patients were established on the spontaneous/timed mode with inspiratory pressures varying from $10-14 \mathrm{~cm} \mathrm{H}_{2} \mathrm{O}$, expiratory at atmospheric, and a respiratory rate of $12-18 / \mathrm{min}$. This allowed triggering of the ventilator while the patient was awake and mandatory ventilation when asleep if the patient failed to breathe spontaneously. Delivery was via a comfortable silicon nasal mask (Medic-Aid Ltd, Sussex) or alternately the Adams' circuit with nasal pillows (Puritan Bennett, Hounslow). The patients adapted to the ventilator during the day for short periods and then used it regularly overnight before discharge home. Parents were taught ventilator management (for example adjustment of controls and cleaning) before discharge. The immediate response to ventilation was assessed by overnight oximetry. We repeated polysomnography after an average of eight months (range 1-24 months) to determine the effectiveness of ventilation but without recording oronasal airflow because of the nasal mask. Although two patients slept with supplemental oxygen at presentation this was not required on ventilation.

\section{FOLLOW UP}

In addition to their routine follow up, patients were contacted personally by the authors $2-6$ years after treatment to determine clinical progress and ventilator usage. Additionally, the authors perused the latest clinical reports.

\section{SLEEP CONTROLS}

The purpose of studying sleep controls was to establish the effect, if any, of hospitalisation on sleep. The controls were therefore studied on the ward under exactly the same circumstances as the hypoxaemic patients. We chose controls who were unlikely to have sleep hypoxaemia. Ten patients (four boys, six girls) attending the Hammersmith Hospital had identical polysomnographic studies on the first or second night of admission. Five had a neuromuscular

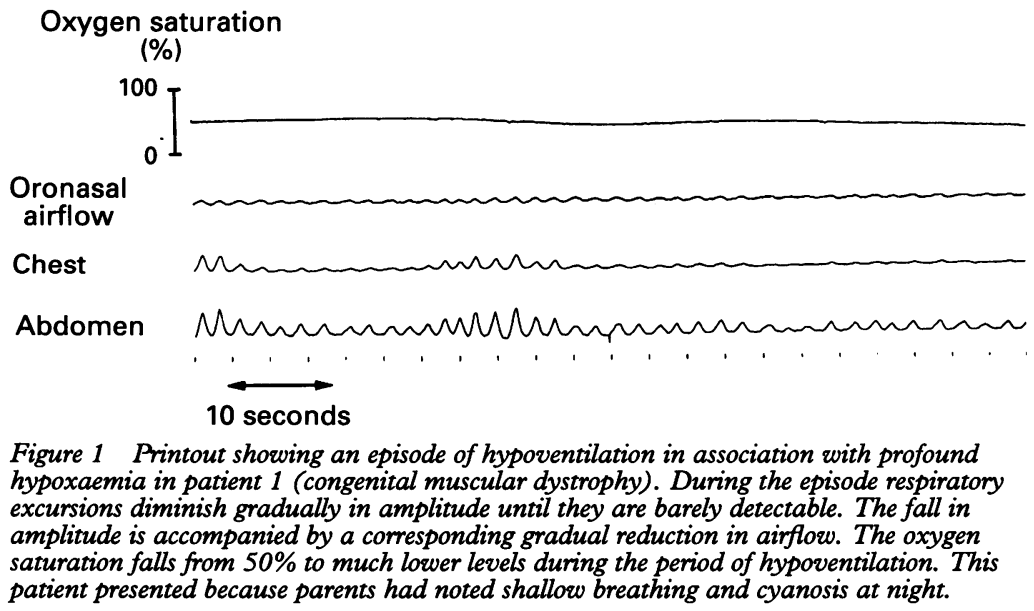

disorder and five a non-neurological disorder. The mean (range) age was $10 \cdot 8(6-22)$ years.

\section{ETHICAL PERMISSION}

This study was approved by the hospital ethical committee. Parents of hypoxaemic patients and control subjects signed informed consent for the sleep studies.

\section{STATISTICS}

Differences in the various sleep parameters before and after introduction of regular nasal ventilation were compared by the paired $t$ test. Patients' diagnostic sleep studies were compared with controls by means of the Student's $t$ test. Statistical analysis was carried out with the Minitab computer program. ${ }^{20}$

\section{Results}

\section{PATIENTS}

The diagnostic sleep study showed that patients were significantly hypoxaemic, spending on average $49 \%$ (range $14-100 \%$ ) of their sleep with their oxygen saturation below $90 \%$ and $19 \%$ (range $0-78 \%$ ) of their sleep with it below $80 \%$. Nasal ventilation was extremely effective in correcting the sleep hypoxaemia with only one patient (6) showing any fall in the oxygen saturation below $90 \%$ (table 1 ). There was no relationship between the severity of the hypoxaemia and the vital capacity, and two patients had a vital capacity of $50 \%$ of expected or more. Daytime blood gases repeated after the introduction of night time ventilation showed resolution of hypoxia in all patients and hypercarbia in five.

The primary respiratory abnormality was hypoventilation (fig 1). On observation of the polysomnographic tracings, the patients did not usually waken during periods of shallow breathing, despite an associated fall in the oxygen saturation.

These periods of hypoventilation occurred repeatedly, could last up to two minutes, and were often complete apnoea. They occurred during deep and REM sleep in all patients except one. The one exception was our youngest patient who had the least amount of REM sleep (patient 5) and was only hypoxaemic during deep sleep. On average 59\% (range $25-80 \%$ ) of the hypoxaemic time was during deep sleep and 33\% (range $0-75 \%$ ) during REM sleep, the proportions of these two phases of sleep that were hypoxaemic being $55 \%$ and $69 \%$ respectively. Two patients ( 1 and 7) were continuously hypoxaemic during all phases of sleep. At referral, two patients ( 7 and 8 ) were on overnight oxygen by nasal cannula which did not correct the hypoventilation episodes nor the daytime hypoxaemia and hypercapnia.

Patients tolerated night time ventilation well. The ventilator was quiet and convenient and five patients have travelled abroad with it. The mean duration of ventilation at follow up has been 3.5 (range 2-6) years. Air swallowing from the nasal mask has not been a prơblem 
Table 2 Sleep parameters; values are mean (range)

\begin{tabular}{lllll}
\hline & $\begin{array}{l}\text { Before } \\
\text { ventilation }\end{array}$ & $\begin{array}{l}\text { After } \\
\text { ventilation }\end{array}$ & $\begin{array}{l}\text { Palue } \\
\text { Value }\end{array}$ & Controls \\
\hline Sleep efficiency (\%) & $91(68-98)$ & $92(80-100)$ & NS & $91(73-99)$ \\
Light sleep (\%) & $15(2-28)$ & $26(10-57)$ & NS & $38(14-76)$ \\
Deep sleep (\%) & $65(37-98)^{\star}$ & $43(14-72)$ & 0.04 & $38(3-70)^{\star}$ \\
REM sleep (\%) & $20(0-54)$ & $30(14-61)$ & NS & $18(1-74)$ \\
Awake (\%) & $8(1-24)$ & $6(<1-19)$ & NS & $5(0-12)$ \\
Total wake shifts in deep sleep & $5(3-8)^{\star \star \star}$ & $<1(0-2)$ & 0.0003 & $1 \cdot 2(0 \cdot 3)^{\star \star \star}$
\end{tabular}

†Comparison of diagnostic sleep study with repeat study on ventilation by paired $t$ test.

${ }^{\star} \mathrm{p}<0 \cdot 02,{ }^{\star \star \star} \mathrm{p}<0 \cdot 001$ : on Student's $t$ test comparing patient's initial diagnostic study with controls.

with maximum airway pressure below $15 \mathrm{~mm}$ $\mathrm{H}_{2} \mathrm{O}$. We found that maximum airway pressure tended to rise during the night as the patients no longer triggered the ventilator and it was important to adjust the controls accordingly. Pressure sores over the bridge of the nose caused some discomfort, and were resolved by alternating the nasal mask with the Adams' circuit nasal pillows. All our patients were able to put on the mask at night independently and could operate the ventilator themselves with little assistance from parents.

Repeat polysomnography on ventilation showed good respiratory excursions throughout the night and periods of spontaneous respiration interspersed with periods of complete reliance on mechanical ventilation. In two patients the mask was dislodged during deep and REM sleep. This was clearly evident by immediate hypoventilation and oxygen desaturation but the patients did not wake (the BiPAP ventilator did not have an alarm system).

All patients reported improved sleep quality on ventilation and resolution of symptoms, such that all would prefer to sleep with the ventilator. Antifailure medication and supplementary oxygen were discontinued. Three patients $(1,6$, and 7$)$ have undergone a general anaesthetic for major scoliosis surgery without any complications and have been weaned back onto regular night time nasal ventilation. The progressive scoliosis in the boy with minicore myopathy (3) was too advanced for surgery.

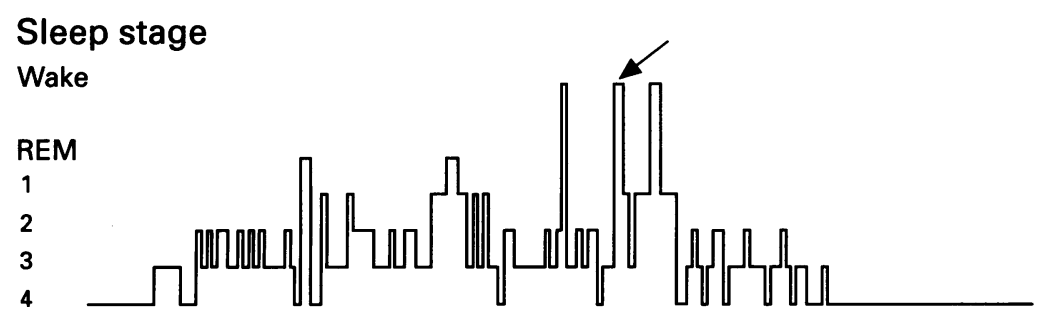

\section{Oxygen saturation}

(\%)

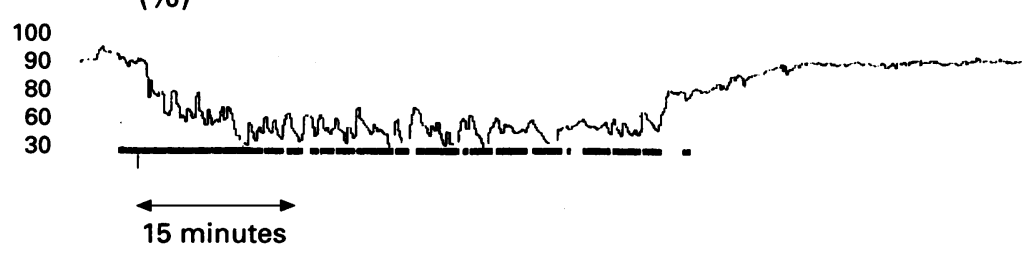

Figure 2 Section of hypnogram, 2 to 4 am, from diagnostic sleep study in patient 3 (minicore disease). Initially he is in stage 4 sleep with oxygen saturation at $90 \%$. This is followed by a period of profound hypoxaemia (oxygen saturation $80 \%$ to $<30 \%$ ) with changes in sleep stages without awakening. After more than 30 minutes of hypoxaemia there are awake epochs (arrow) from stage 3 and 4 sleep (deep sleep).
Later follow up confirmed that all patients were continuing to use the ventilator every night. Seven out of eight patients had started full time education. Symptoms of morning headaches and poor appetite had resolved and all patients had gained weight. Four patients were on the 50th centile for weight, two patients were on the 25 th centile, and two on the 3rd centile. In the years after ventilation there have been no admissions for chest infections.

Sleep architecture was normally preserved both before treatment and subsequently. Hypoxaemic patients had significantly more deep sleep than controls and this fell on ventilation. They also had significantly more wake shifts in deep sleep, than did controls (table 2). The longest duration of these wake shifts was six minutes. The number of wake shifts fell significantly on nasal ventilation but this fall was not related to the fall in amount of deep sleep on ventilation (table 2). Neither the amount of deep sleep not the number of wake shifts in deep sleep related to the severity of the hypoxaemia. The hypnogram in three patients showed deep sleep wake epochs during hypoxaemia, but other patients did not show this relationship (fig 2 ).

\section{SLEEP CONTROLS}

In the sleep control subjects the percentage of time spent in REM sleep, sleep efficiency, and the amount of time spent awake after the onset of sleep were comparable with age matched normal data. ${ }^{1521}$ None had hypoxaemia (oxygen saturation $<90 \%$ ) on their polysomnographic night.

\section{Discussion}

Although the occurrence of sleep hypoventilation in congenital muscle disorders is well recognised, ${ }^{1-5}$ there is probably little awareness of the frequency of respiratory failure in mildly disabled patients and the effectiveness of night time nasal ventilation. ${ }^{361011}$ This series of eight patients demonstrates the need for a greater awareness of abnormal sleep related breathing patterns. The patients presented with recurrent respiratory infections, poor appetite and weight gain, and a history of headaches or acute-on-chronic respiratory failure, and it seemed likely that sleep hypoventilation had been a problem for a significant period beforehand. It is noteworthy that two referrals were based on parents' observations while the patients themselves were unaware of any breathing difficulties. In general the significance of symptoms was not fully appreciated, except retrospectively once ventilation was started.

Failure to thrive has been previously associated with sleep hypoxaemia due to tonsillar obstruction and, as in our patients, resolution of hypoxaemia was associated with weight gain. 22 Ideally sleep hypoxaemia should be identified at an early stage, that is before the occurrence of acute decompensation during intercurrent infection, the development of chronic respiratory failure, or deterioration 
of respiratory function associated with progressive scoliosis. Scoliosis is a frequently associated feature reflecting the axial distribution of muscle weakness and it is essential to diagnose any respiratory problems before scoliosis surgery.

The risk of acute respiratory failure does not correlate with the severity of night time hypoxaemia. This is not surprising as acute respiratory failure is usually precipitated by infection and factors relating to infection such as change in upper airways resistance and lung compliance. Nevertheless, we have not yet seen acute respiratory failure without some suggestion of prior sleep hypoventilation. There is also a poor correlation between the severity of the sleep hypoxaemia and the reported symptoms. The perception of symptoms may relate to the age of the child and the chronicity of the hypoxaemia.

To diagnose nocturnal hypoxaemia at an early stage requires regular careful clinical examination and overnight sleep study when indicated. Clinical assessment should include evidence of abnormal respiratory muscle activity such as abdominal 'squeeze' and paradoxical breathing. The former suggests a small tidal volume ${ }^{3}$ and the latter a weak diaphragm. ${ }^{23}$ Patients with severe weakness of neck flexors and a rigid spine are also particularly liable to respiratory failure even though ambulant. ${ }^{311}$ The sleep study may be by oximetry alone but more detailed information is often useful. Oximetry with respiratory channels, that is inductance plethysmography and airflow, overcomes the problem of poor signal artifacts from a dislodged oximeter probe, is simple to perform, and does not require a great deal of expertise to interpret and is practical even in young children. Eight channel polysomnography, if available, is more definitive, and allows good comparative sequential analysis and planned intervention. We believe that sequential sleep studies, whatever the method, should be routinely done in ambulant children with certain neuromuscular diseases - namely minicore, nemaline and 'minimal change' myopathies, congenital muscular dystrophy, and the rigid spine syndrome. Such studies should be considered as an essential part of a fully respiratory assessment, particularly as standard lung function tests such as vital capacity do not seem to reliably predict sleep hypoventilation.

The decision to provide regular night time ventilation is balanced between the patient's overall disability and the additional burden of the technique of ventilation. ${ }^{24}$ In our series, all patients were ambulant and had relatively mild physical disability and generally a good prognosis.

Nasal ventilation corrected the sleep hypoventilation and reversed the progression of chronic respiratory failure without imposing a major burden. Additionally, ventilation reduced the frequency of debilitating chest infections and made spinal stabilising surgery possible in three patients. The age of the patient is a critical factor as the youngest that we and others have successfully established on nasal ventilation has been at 6 years of age. ${ }^{25}$
Therapeutic options for younger patients are limited to cuirass or tank ventilation or a tracheotomy. ${ }^{26}$ The underlying myopathies described in our patients are not generally progressive but there should be some caution in advocating ventilation when scoliosis is not amenable to surgery.

Sleep was remarkably well preserved despite profound hypoxaemia. There was normal progression through the sleep stages, and sleep efficiency and total sleep time were within the normal range. Most patients had REM sleep. This preservation presumably explains why patients had so few symptoms. Nevertheless all patients subjectively noted improved sleep and well being on ventilation. This is consistent with previous experience. 10112728

The sleep disturbance at the diagnostic sleep study was relatively subtle and confined to excess deep sleep with increased 30 second wake shifts. It would have been interesting to have looked at microarousals - that is, two seconds of alpha rhythm, which have been reported to be increased in hypoxaemia. ${ }^{29}$ Microarousals are likely to be increased if wake shifts of 30 second epochs, reflecting a stage in the sleep/wake cycle also containing alpha rhythm, were increased. ${ }^{16}$ Increase number of wake shifts is a previously documented measure of poor sleep quality. ${ }^{16} 3031$

Our patients showed excess deep sleep in contrast to previous studies of hypoxaemia, in children and adults, which have shown deep sleep to be reduced. ${ }^{32} 33$ Other reports of hypoxaemia resulting from hypoventilation in neuromuscular disorders show an association with deep sleep. ${ }^{35-37}$ In contrast obstructive sleep apnoea in Duchenne muscular dystrophy, ${ }^{38} 39$ and in non-neuromuscular disorders, ${ }^{28} 29$ is associated with, and often confined to, REM sleep. Hypoventilation during deep sleep probably has a different pathophysiology from obstructive apnoeas during REM sleep.

In two patients, the ventilator disconnected, causing profound hypoxaemia, but they did not wake. Although hypoxaemia is known to trigger awakening, the factors causing this are not clearly understood. ${ }^{28}$ In addition, deep sleep may intrinsically be associated with an increased awakening threshold. ${ }^{34} \mathrm{~A}$ ventilator with an alarm system is an advantage.

In conclusion, we have shown that mildly disabled children with certain types of muscle disease can have significantly potentially life threatening sleep hypoventilation. The significance of clinical symptoms may not be fully appreciated or there may be no apparent symptoms. In view of the chronic debility, and the risk of acute respiratory failure, cor pulmonale and complications of spinal surgery, early diagnosis is desirable, if not mandatory. There should be clinical vigilance with a careful respiratory history, examination for abnormal respiratory muscle activity, measurement of vital capacity, and sequential sleep studies. As nasal ventilation is an effective and noninvasive mode of treatment the decision to intervene is readily justified. 
This project was supported by a research grant from the Muscular Dystrophy Group of Great Britain. In addition financial support was received from the Handicapped Children's Aid Committee. The authors are grateful to Dr A Sharply for he comments and Dr M White for providing additional information on a patient. We thank Dr Hopp for the sleep study data on ventilation on two of the patients, done on similar equipment

1 Riley DJ, Danielle RP, Edelman NH. Blunted respiratory drive in congenital myopathy. $A m \mathcal{F}$ Med 1977; 63: 459-65.

2 Maayan $\dot{C}$, Springer C, Armon Y, Bar-Yishay E, Shapira Y, Godfrey S. Nemaline myopathy as a cause of sleep Y, Godfrey S. Nemaline myopathy as a caus
hypoventilation. Pediatrics 1986; 77: 390-5.

3 Heckmatt JZ, Loh L, Dubowitz V. Nocturnal hypoventilation in children with non-progressive neuromuscula disease. Pediatrics 1989; 83: 250-5.

4 Bye P, Ellis E, Issa F, Donnelly P, Sullivan C. Respiratory failure and sleep in neuromuscular disease. Thorax 1990 45: 241-7.

5 Kawata A, Suga M, Miyamoto K, Hirose K, Tanabe H. Rigid spine and nocturnal alveolar hypoventilation. Intern Med 1993; 32: 638-40.

6 Ellis E, Bye P, Bruderer J, Sullivan C. Treatment of respiratory failure during sleep in patients with neuromuscular disease; positive-pressure ventilation through as nose mask. Am Rev Respir Dis 1987; 135: 148-52.

7 Bach JR, Alba A, Mosher R, Delaubier A. Intermittent positive pressure ventilation via nasal access in the managetive pressure ventilation via nasal access in the manage-
ment of respiratory insufficiency. Chest 1987; 92: 168-70.

ment of respiratory insufficiency. Chest 1987 ; 92: 168-70.
8 Bach J, Alba A. Management of chronic alveolar hypoventilation by nasal ventilation. Chest 1990; 97: 52-7.

9 Carroll N, Branthwaite M. Control of nocturnal hypoventilation by nasal intermittent positive pressure ventilation. Thorax 1988; 43: 349-53.

10 Kerby GR, Mayer LS, Pingleton S. Nocturnal positive pressure ventilation via nasal mask. Am Rev Respir Dis 1987; 135: 738-40.

11 Heckmatt J, Loh L, Dubowitz V. Night-time nasal ventilation in neuromuscular disease. Lancet $1990 ; 335$ : 659-82.

12 Luque E. Segmental spinal instrumentation for correction of scoliosis. Clin Orthop 1982; 163: 192-8.

13 Godfrey S, Kamburoft P, Nairn J, et al. Spirometry, lung volumes and airway resistance in normal children aged 5-18 years. British fournal of Diseases of the Chest 1970; 64: 15-24.

14 Carskadon $M$, Dement W. Normal human sleep: an overview. In: Kryger $M$, Roth T, Dement W, eds. Principles and practice of sleep medicine. Philadelphia: WB Principles and practice of

15 Coble P, Kupfer D, Taska L, Kane J. EEG sleep of normal healthy children. Par 1: Findings using standard measurement methods. Sleep 1984; 7: 289-303.

16 Carskadon M, Brown E, Dement W. Sleep fragmentation in the elderly: relationship to daytime sleep tendency Neurobiol Aging 1982; 3: 321-7.

17 Rechtschaffen A, Kales A. A manual of standardised terminology, techniques, and scoring system for sleep states in human subjects. Washington DC: US Government Printing Office, Public Health Service, 1968: 1-12.

18 Baker TL. Introduction to sleep and sleep disorders. Symposium of sleep apnoea disorders. Med Clin North Am 1985; 69: 1123-52.

19 Cherniack N. Respiratory dysrhythmias during sleep. N Engl f Med 1981; 305: 325-30.
20 Minitab Inc. Minitab computer programme. Philadelphia: Minitab Inc.

21 Palm L, Persson E, Elmqvist D, Blennow G. Sleep and wakefulness in normal preadolescent children. Sleep 1989; 12: 299-308.

22 Stradling JR, Thomas G, Warley ARH, Williams $P$ Freeland A. Effect of adenotonsillectomy on nocturnal hypoxaemia, sleep disturbance, and symptoms in snoring children. Lancet 1990; 335: 249-53.

23 Newsom Davis J, Goldman M, Loh L, Casson M Diaphragm function and alveolar hypoventilation. Qf Med 1976; 45: 87-100.

24 American Thoracic Society. Home mechanical ventilation of pediatric patients. Am Rev Respir Dis 1990; 141: of pediatr $258-9$.

25 Ellis E, Mccauley V, Mellis C, Sullivan C. Treatment of alveolar hypoventilation in a six-year-old girl with intermittent positive pressure ventilation through a nose mask. Am Rev Respir Dis 1987; 136: 188-91.

26 O'Leary J, King R, Leblanc M, Moss R. Cuirass ventilation in childhood neuromuscular disease. $\mathcal{F}$ Pediatr 1979; 94 419-21.

27 Sullivan C, Berthon Jones M, Issa F. Reversal of obstructive sleep apnoea by positive airway pressure applied through the nares. Lancet $1981 ;$ i: $862-5$.

28 Stradling J. Disorders of ventilatory control and sleep apnoea syndromes. In: Brewis R, Gibson $G$, Geddes $B$ eds. Respiratory medicine. London: Balliere Tindall, 1990 1352-72.

29 Fleetham F, West P, Mezon B, Conway W, Roth T, Kryger $M$. Sleep, arousals, and oxygen desaturation in chronic obstructive pulmonary disease. Am Rev Respir Dis 1982; 126: 429-33.

30 Avital A, Steljes D, Pasterkamp H, Kryger M, Sanchez I, Chernick V. Sleep quality in children with asthma treated with theophylline or cromolyn sodium. F Pediatr 1991, 119: 979-84.

31 Levine B, Roehrs T, Stepanski E, Zorick F, Roth T Fragmenting sleep diminished its recuperative value. Sleep 1987; 10: 590-9.

32 Spiegel $\mathbf{R}$. Sleep and sleeplessness in advanced age. In Weitzman E, ed. Advances in sleep research. Vol 5 . Lancaster: MTB Press, 1981: 43-60.

33 Guilleminault C. Obstructive sleep apnoea syndrome in children. In: Guilleminault C, ed. Sleep and its disorders in children. New York: Raven Press, 1987: 213-24.

34 Kryger M, Steljes D, Yee W-C, Mate E, Smith SA Mahowald M. Central sleep apnoea in congenital muscular dystrophy. $¥$ Neurol Neurosurg Psychiatry 1991; 54 cular dyst

35 Gilmartin J, Cooper B, Griffiths C, et al. Breathing during sleep in patients with myotonic dystrophy and nonmyotonic respiratory muscle weakness. $Q \mathcal{F}$ Med $1991 ; 78$ 21-31.

36 Coccagna G, Mantovani M, Parchi C, Mironi F, Lugaresi E. Alveolar hypoventilation and hypersomnia in myotonic dystrophy. $\mathcal{f}$ Neurol Neurosurg Psychiatry 1975; 38 977-84.

37 Smith P, Calverley P, Edwards R. Hypoxaemia during sleep in Duchenne muscular dystrophy. Am Rev Respir Dis 1988; 137: 884-8.

38 Khan Y, Heckmatt J. Obstructive apnoeas in Duchenne muscular dystrophy. Thorax 1994; 49: 157-61.

39 Dagan Y, Lavie P, Bleich A. Elevated awakening thresholds in sleep stage 3-4 in war-related post-traumatic stress disorder. Biol Psychiatry 1991; 30: 618-22. 